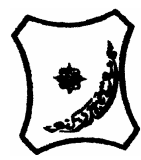

Bayero Journal of Pure and Applied Sciences, 9(2): 154 - 158

Received: April, 2016

Accepted: August, 2016

ISSN $2006-6996$

\title{
ANALYSIS OF AURORAL ELECTROJET MAGNETIC INDICES
}

\author{
${ }^{1}$ Tijjani, B.I., ${ }^{1}$ Mijinyawa, U.M., ${ }^{2}$ Bala, S. and ${ }^{1 *}$ Galadanci, G. S. M. \\ ${ }^{1}$ Department of Physics, Bayero University, Kano. NIGERIA \\ ${ }^{2}$ Department of Mathematical Sciences, Bayero University, Kano. NIGERIA \\ Email: ${ }^{1}$ idrithtijjani@gmail.com, ${ }^{1 *}$ gsmgalad@gmail.com \\ ${ }_{1 *}$ Corresponding author. Tel: 08037260380
}

\section{ABSTRACT}

In this paper magnetic indices were extracted from World Data Center (WDC) for geomagnetism forsix years (2008 to 2013). The indices extracted are AuroralElectrojet (AE), Auroral Upper (AU), Auroral Lower (AL) and Auroral Oval (AO). The indices daily values measured at 24 hours Universal Time (UT) were averaged to monthly values. Time series statistical analysis was carried out on the monthly averaged data using SPSS version 16.0 with expert modeler, to determine the level, trend and seasonal variations of the indices.From the results obtained after the statistical analysis, it can be seen that the indices have seasonal patterns of which AE, AL and AO as March but AU has July. $A / s o$ from the values of the coefficient of determination, $R^{2}$, it can also be concluded that $A E$ and $A L$ can be better predicted from the models than $A U$ and $A O$. From the results obtained, simple seasonal model for $A E, A L$, and $A O$, and winter's additive for $A U$ was determined. Using the values of $R^{2}$, it can be seen that the models for $A E$ and $A L$ can be used to predict or make forecast of the behavior of the indices. It was also discovered that level (alpha) has more significant contributions in the behavior of the system than seasonal.

Keywords: Magnetic indices, World Data Center, Auroral, Level, Trend, Season, Expert modeler.

\section{INTRODUCTION}

The AuroralElectrojet is an enhanced electric current in the polar ionosphere associated with charged particle precipitation and field aligned currents during substorms (Chen et al., 2003). A substorm sometimes referred to as a magnetosphericsubstorm or an Auroralsubstorm is a brief disturbance in the Earth's magnetosphere that causes energy to be released from the 'tail' of the magnetosphere and injected into the high latitude ionosphere. Visually, a substorm is seen as a sudden brightening and increased movement of auroral arcs.

In the northern hemisphere, the bright lights observed are called Aurora Borealis (or northern lights) and Aurora Australis in the southern hemisphere. Substorms were first described by the Norwegian scientist Kristian Birkelandin 1908, which he called polar elementary storms. Sydney Chapman used the term substorm about 1960 which is now the standard term. The characteristics of a substorm was first described by a Japanese geophysicist named Syun-IchiAkasofu in 1964 (Sarris and Li, 2005).

Substorms are distinct from geomagnetic storms in that; the latter take place over a period of several days, are observable from anywhere on Earth, inject a large number of ions into the outer radiation belt, and occur once or twice a month during the maximum of the solar cycle and a few times a year during solar minimum. Substorms, on the other hand, take place over a period of few hours, are observable primarily at the Polar Regions, do not inject many particles into the radiation belt, and are relatively frequent, often occurring only a few hours apart from each other. Substorm occurrence becomes more frequent during a geomagnetic storm when one substorm may start before the previous one has completed (Potemra, 1991). The source of the magnetic disturbances observed at the Earth's surface during geomagnetic storms is the ring current, whereas the sources of magnetic disturbances observed on the ground during substorms are electric currents in the ionosphere at high latitudes (Stern and Peredo, 2001).

Substorms can cause magnetic field disturbances in the auroral zones up to a magnitude of $1000 \mathrm{nT}$, roughly $2 \%$ of the total magnetic field strength in that region. The disturbance is much greater in space, as some geosynchronous satellites have registered the magnetic field dropping to half of its normal strength during a substorm. The most visible indication of a substorm is an increase in the intensity and size of polar auroras (Stem and Peredo, 2001). Substorms occur roughly six times per day, though they are more intense and more frequent during a geomagnetic storm.

In 2012, during Substorm monitoring activity named THEMIS, the observation of the dynamics of a rapidly developing substorm, confirmed the existence of giant magnetic ropes and small explosions in the outskirts of Earth's magnetic field. (NASA, 2012). The geomagnetic field strongly impacts society. During magnetic storms which can be identified by large decrease of the geomagnetic field strength in low latitudes, the radiation belt is developed, causing frequent malfunction of man-made satellites. When the polar geomagnetic field is disturbed strongly; that is, when substorms take place, an electric current is induced over oil pipe lines and high-voltage power lines, and when it happens then such infrastructures are damaged severely. 
In order to predict such phenomena, research related to 'space weather forecast' is in progress in most developed countries. Geomagnetic field research is an important factor in our daily lives.

An extensive study of auroras showed that the auroral oval expands both equatorward and poleward during magnetic substorms (Akosofu, 1972). Kamide and Matsushita (1979a, b) calculated the ionospheric current system caused by field-aligned currents, and showed that the current pattern in the polar region, especially the electrojet, strongly depends upon the distribution of the field-aligned currents and ionospheric conductivity.

In this paper, the magnetic indices $A E, A U, A L$ and $A O$ were extracted from World data center for geomagnetism for six years (2008 to 2013). The AE indices were derived from geomagnetic variations in the horizontal component observed at selected (1013) observatories along the auroral zone in the northern hemisphere. Then among the data from all the stations at each given time (UT), the largest and smallest values are selected. The $A U$ and $A L$ indices are respectively defined by the largest and the smallest values so selected. The symbols, $A U$ and $A L$, derive from the fact that these values form the upper and lower envelopes of the superposed plots of all the data from these stations as functions of UT. The $A E$ and $\mathrm{AO}$ indices are defined as:

$$
\begin{aligned}
& \mathrm{AE}=\mathrm{AU}-\mathrm{AL} \\
& A O=\frac{A U+A L}{(2)}
\end{aligned}
$$

The term "AE indices" is usually used to represent these four indices ( $A U, A L, A E$ and $A O)$. The $A U$ and $A L$ indices are intended to express the strongest current intensity of the eastward and westward auroralelectrojets, respectively. The $\mathrm{AE}$ index represents the overall activity of the electrojets, and the $A O$ index provides a measure of the equivalent zonal current. The data were then statistically analysed using time series analysis with SPSS version 16.0, using expert modeler. A forecast for the year 2014 was also made.

\section{METHODOLOGY}

The area covered by this work is twelve observatories listed in the table below. Table 1: List of AE (12) Stations

\begin{tabular}{lccccc}
\hline OBSERVATORY & IAGA CODE & \multicolumn{2}{c}{ GEOGRAPHIC COORD. } & \multicolumn{2}{c}{ GEOMAGNETIC COORD. } \\
& & LAT. $\left({ }^{0} \mathrm{~N}\right)$ & LONG. $\left({ }^{0} \mathrm{E}\right)$ & LAT. $\left({ }^{0} \mathrm{~N}\right)$ & LONG. $\left({ }^{0} \mathrm{E}\right)$ \\
\hline ABISKO & ABK & 68.36 & 18.82 & 66.04 & 115.08 \\
DIXON ISLAND & DIK & 73.55 & 80.57 & 63.02 & 161.57 \\
CAPE CHELYUSKIN & CCS & 77.72 & 104.28 & 66.26 & 176.46 \\
TIXIE BAY & TIK & 71.58 & 129.00 & 60.44 & 191.41 \\
CAPE WELLEN & CWE & 66.17 & 190.17 & 61.79 & 237.10 \\
BARROW & BRW & 71.30 & 203.25 & 68.54 & 241.15 \\
COLLEGE & CMO & 64.87 & 212.17 & 64.63 & 256.52 \\
YELLOWKNIFE & YKC & 62.40 & 245.60 & 69.00 & 292.80 \\
FORT CHURCHILL & FCC & 58.80 & 265.90 & 68.70 & 322.77 \\
POSTE-DE-LA-BALEINE & PBQ & 55.27 & 282.22 & 66.58 & 347.36 \\
NARSARSUAQ & NAQ & 61.20 & 314.16 & 71.21 & 36.79 \\
(NARSSARSSUAQ) & & & & & 71.04 \\
LEIRVOGUR & LRV & 64.18 & 338.30 & 70.22 & 7 \\
\hline
\end{tabular}

The magnetic indices extracted from World Data Center (WDC) for geomagnetism for six years (2008 to 2013) were averaged to monthly values. Time series statistical analysis was carried out on the monthly averaged data using SPSS 16.0 with expert modeler, to determine the level, trend and seasonal variations of the indices. The expert modeler determined whether ARIMA (Auto Regression Integration Moving Average) or exponential smoothing is the best based on which model gives the highest $\mathrm{R}^{2}$.

\section{RESULTS AND DISCUSSIONS}

Figure 1 shows the plots for the real and the predicted values. From the results obtained it shows that the data activities have no trend, but level and seasonal factors. From the plot of the predicted $A E$, it can be observed that the most prominent seasonal pattern is in march of each year. The value of $R^{2}$ implies that about $68.07 \%$ of the variation is explained by the dependent variable, and its corresponding $\mathrm{p}$-value being greater than 0.05 means that the data does not significantly fit the model.

From the model parameters, the value of $p$-value for the alpha signifies that the level is a very significant predictor in the model. For the seasonal parameter, the value of its $p$-value implies that season is not a significant predictor in the model. 


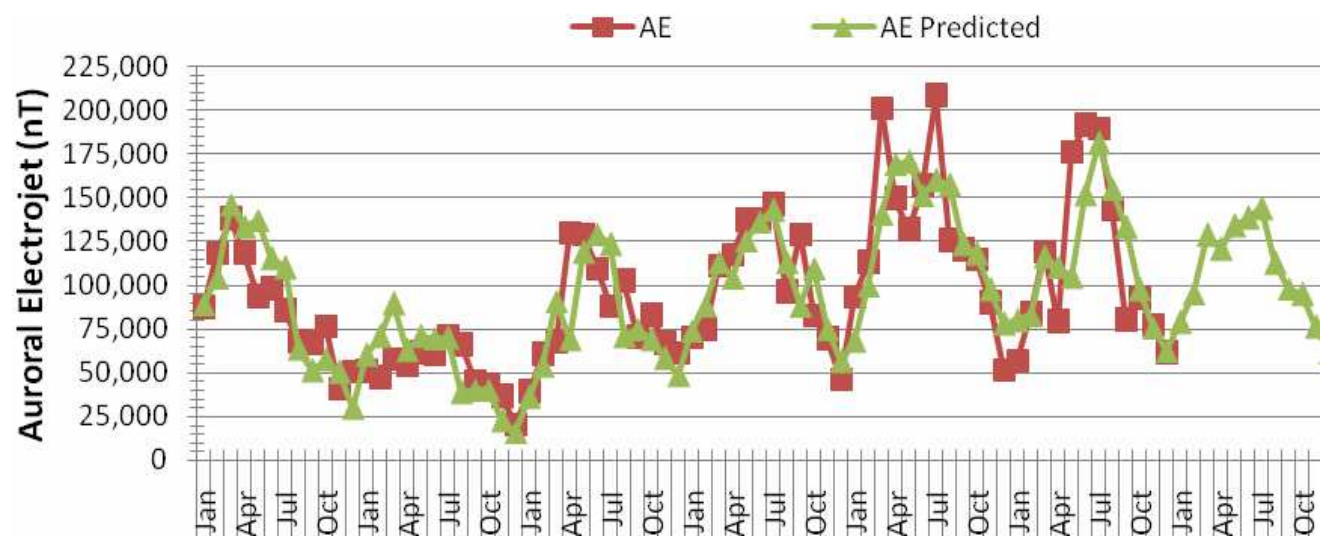

Figure 1: Plots of Auroralelectrojet for the real and the predicted data.

Model Type: Simple Seasonal,No Transformation: $\mathrm{R}^{2}=0.6807, \mathrm{Sig}=0.3271$

Exponential Smoothing Model Parameters: Alpha (Level)=0.5999, Sig=1.39E-06, Delta (Season)= 5.83E-05, Sig $=0.9997$.

Figure 2 shows the plots of the real and the predicted data. From the model parameters obtained, it shows that the activities have a linear trend and a seasonal effect that does not depend on the level of the series. Its smoothing parameters are level, trend, and season. From the predicted $A U$, it can be observe that the most prominent seasonal pattern occurs in July of each year.

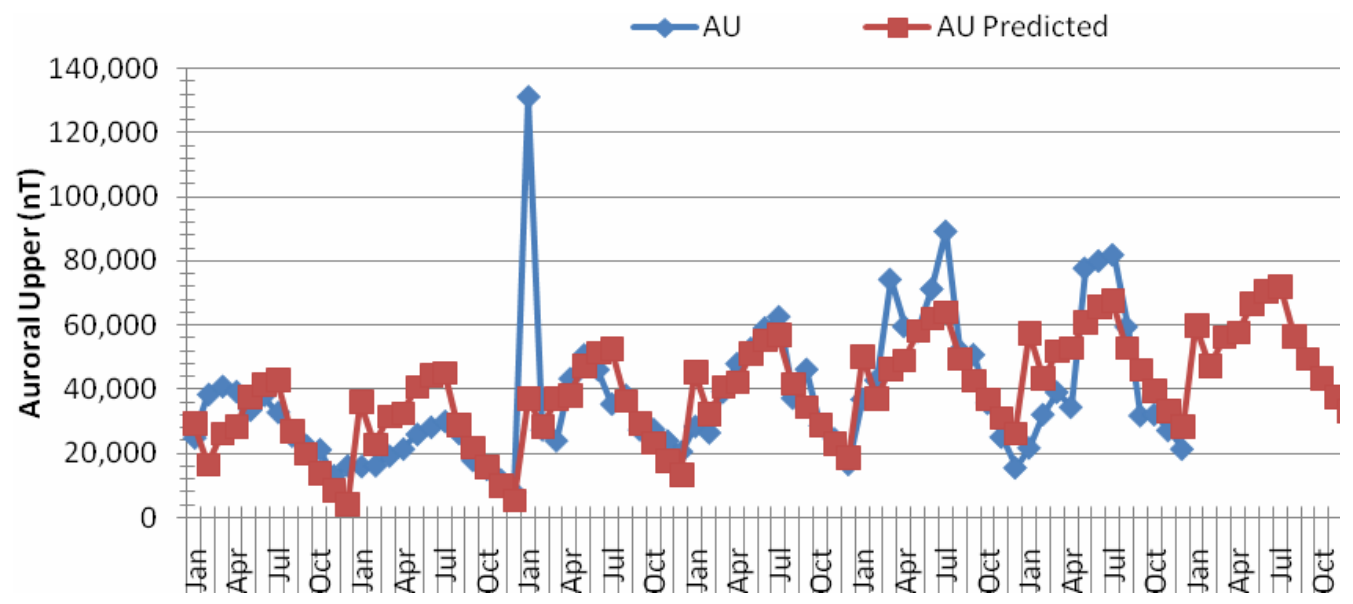

Figure 2: Plots of Auroral Upper for the real and the predicted data.

Model Type: Winters' Additive, No Transformation: $\mathrm{R}^{2}=0.451154286, \mathrm{Sig}=0.486827$

Exponential Smoothing Model Parameters: Alpha (Level)=0.041 Sig=.177, Gamma (Trend)= 3.450E-6, Sig=.999, Delta (Season) $=0.001$, Sig=.992.

From the value of $\mathrm{R}^{2}$ it implies that $45.12 \%$ of the variation is explained by the dependent variable, and its corresponding $\mathrm{p}$-value means that the data does not significantly fit the model.

From the model parameters, the $p$-value of the alpha signifies that level is not a significant predictor in the model. The value of $p$-value for the gamma, implies that gamma is not a significant predictor in the model.
Figure 3 shows the plots of the real and predicted data. From the results obtained from SPSS it shows that the data activities have no trend, but level and seasonal factors. That is its smoothing parameters are level and season. From the plot of the predicted $A E$, it can be observed that the most prominent seasonal pattern is in March of each year 


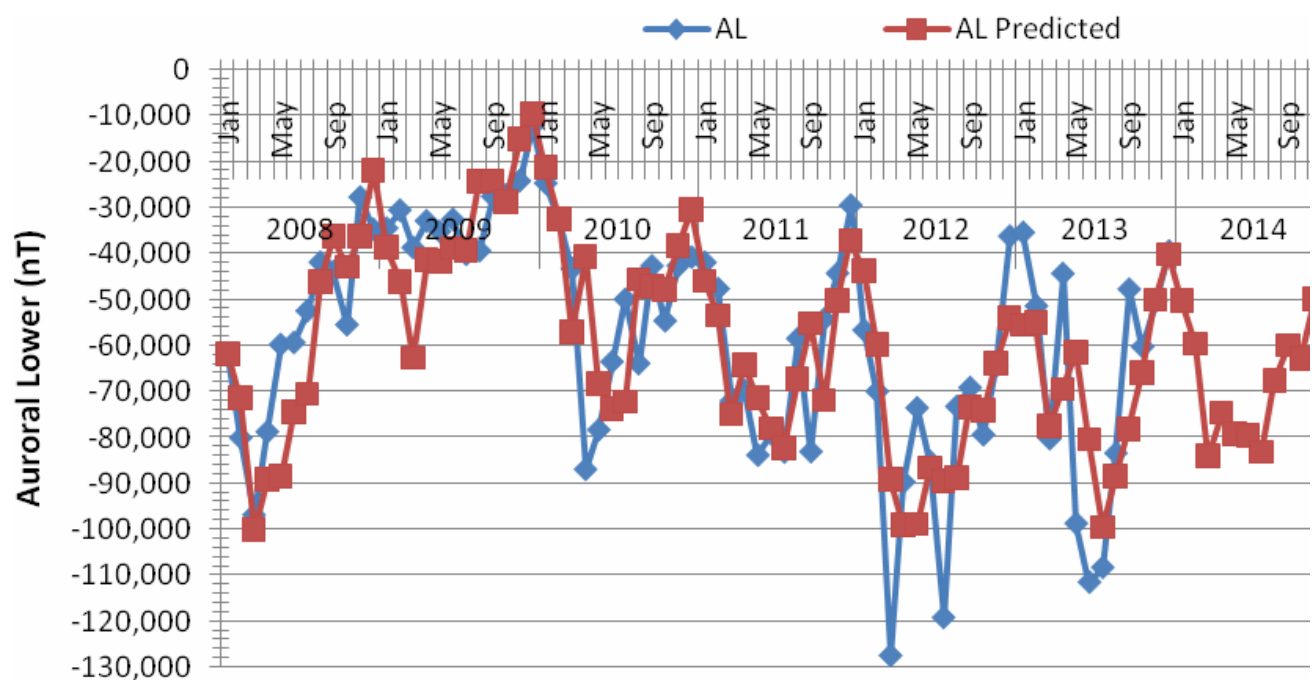

Figure 3: Plots of Auroral Lower for the real and the predicted data.

Model Type: Simple Seasonal, No Transformation: $R^{2}=0.611195804, \operatorname{Sig}=0.281193$

Exponential Smoothing Model Parameters: Alpha (Level)=0.500, Sig=1.19E-5; Delta (Season)= 4.61E-5, Sig $=0.9997$

The value of $\mathrm{R}^{2}$ implies that $61.12 \%$ of the variation is explained by the dependent variable, and the $\mathrm{p}$-value shows that the data do significantly fit the model.

Figure 4 shows the plots for the real and predicted data of the AO. From the results obtained from SPSS it shows that the data activities have no trend, but From the model parameters, the $p$-value of the alpha implies that level is a very significant predictor in the model, and the p-value of the season means that season is not a significant predictor in the model. level and seasonal factors. That is its smoothing parameters are level and season. From the plot of the predicted $A E$, it can be observed that the most prominent seasonal pattern is in march of each year.

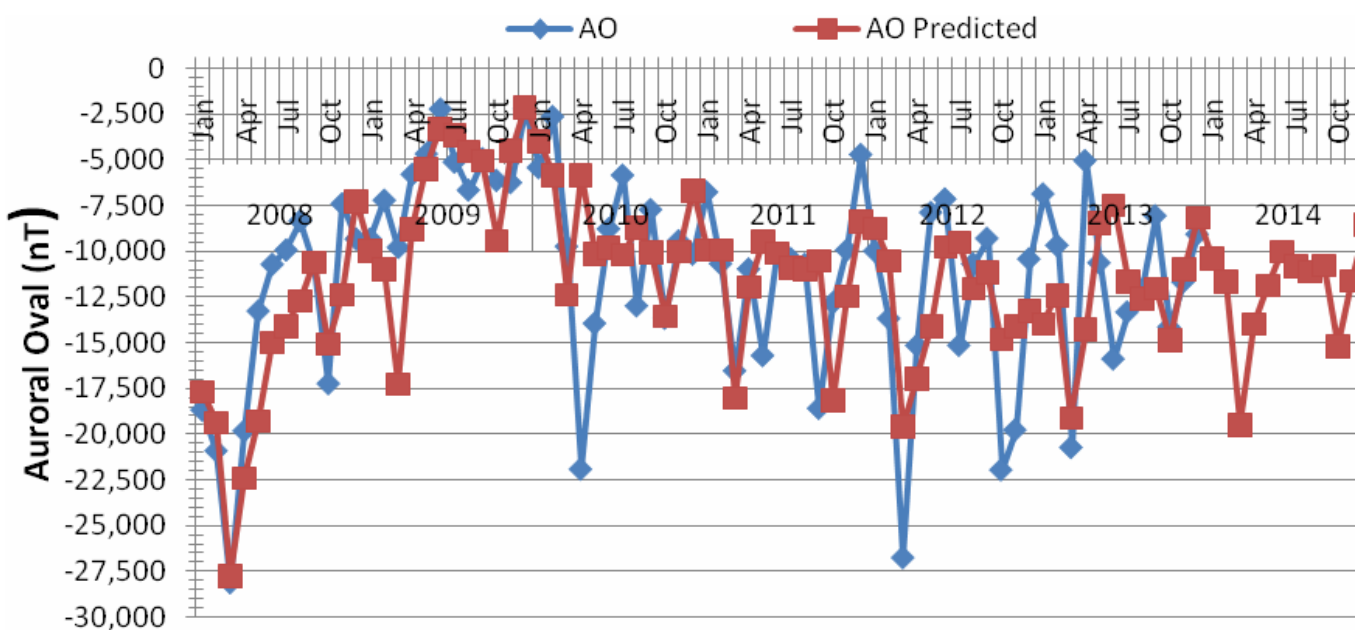

Figure 4: : Plots of Auroral Oval for the real and the predicted data.

Model Type: Simple Seasonal, No Transformation: $\mathrm{R}^{2}=0.4251, \mathrm{Sig}=0.1188$

Exponential Smoothing Model Parameters: Alpha (Level)=0.4000, Sig=1.4410E-4; Delta (Season)=4.13E-5, Sig $=0.9998$.

The value of $\mathrm{R}^{2}$ implies that $42.51 \%$ of the variation is explained by the dependent variable, and its corresponding $p$-value shows that the data do not significantly fit the model.

From the model parameters, the p-value of the alpha shows that level is a very important factor in the prediction of the model, while the p-value of the season shows that seasonal factor is not a significant predictor in the model. 


\section{CONCLUSION}

From the results obtained after the statistical analysis, it can be seen that the indices have seasonal patterns of which $A E, A L$ and $A O$ as March but $A U$ has July. Also from the values of $R^{2}$, it can also be concluded that $A E$ and $A L$ can be better predicted from the models than AU and AO. From the statistical analysis conducted, it is discovered that the best model that describes these indices is simple seasonal, and the best two models that best described their behaviors are $\mathrm{AL}$ and $\mathrm{AE}$. From these two models it can be observed that the most important parameters in the

\section{REFERENCES}

Akasofu, S.I.(1972). Magnetosphericsubstorms: A model, in Solar-Terrestrial Physics, Part 3, edited by E. R. Dyer, 131 pp., D. Reidel, Norwell, Mass.

Chen, Geng-Xiong Wen-Yao Xu1, Zi-Gang Wei, B.H.Ahn and Y. Kamide (2003).Auroralelectrojet oval.Earth Planets Space, 55, 255-261,

Kamide, Y. and S. Matsushita (1979a). Simulation studies of ionospheric electric fields and currents in relation to field-aligned currents, 1. Quiet periods, J. Geophys. Res., 84, 4083-4098

Kamide, Y. and S. Matsushita (1979b). Simulation studies of ionospheric electric fields and currents in relation to field-aligned currents, models are levels. The seasonal parameters do not have much significant contributions. The results also shows how magnetic indices can be models using times series analysis. However, the problem with the proposed models is that they cannot be used to predict substorms and geomagnetic storms.

\section{Acknowledgement}

The authors wish to acknowledge the world data centre (WDL) from where te magnetic indices were ectracted from.

2. Substorms, J. Geophys. Res., 84, 40994115

NASA (2012) Spacecraft Make New Discoveries About Northern Lightshttp://www.nasa.gov/mission_pages/t hemis/auroras/northern_lights.html

Potemra, T. (1991).MagnetosphericSubstorms. Washington, D.C.: Am. Geophysical Union. p.488.ISBN0-87590-030-5.

Sarris, T. and Li, X. (2005).Evolution of the Dispersionless Injection Boundary associated with Substorms. AnnalesGeophysicae23: 877-884. Bibcode: 2005AnGeo..23..877S. doi:10.5194/angeo-23-877-2005.

Stern, David P. and Peredo, Mauricio (2001).Substorms. Southwest Research Institute. Retrieved online, 24 March, 2010. 